\title{
On the characteristic polynomial of $n$-Cayley digraphs
}

\author{
Majid Arezoomand \\ Department of Mathematical Sciences \\ Isfahan University of Technology \\ Isfahan, Iran \\ arezoomand@math.iut.ac.ir
}

\author{
Bijan Taeri \\ Department of Mathematical Sciences \\ Isfahan University of Technology \\ Isfahan, Iran \\ b.taeri@cc.iut.ac.ir
}

Submitted: Jan 31, 2013; Accepted: Sep 24, 2013; Published: Oct 7, 2013

Mathematics Subject Classifications: 05C50, 05C25, 05C31

\begin{abstract}
A digraph $\Gamma$ is called $n$-Cayley digraph over a group $G$, if there exists a semiregular subgroup $R_{G}$ of $\operatorname{Aut}(\Gamma)$ isomorphic to $G$ with $n$ orbits. In this paper, we represent the adjacency matrix of $\Gamma$ as a diagonal block matrix in terms of irreducible representations of $G$ and determine its characteristic polynomial. As corollaries of this result we find: the spectrum of semi-Cayley graphs over abelian groups, a relation between the characteristic polynomial of an $n$-Cayley graph and its complement, and the spectrum of Cayley graphs over groups having cyclic subgroups. Finally we determine the eigenspace of $n$-Cayley digraphs and their main eigenvalues.
\end{abstract}

Keywords: $n$-Cayley digraph; linear representations of groups; characteristic polynomial

\section{Introduction}

Graphs come in two principle types: directed graphs and undirected graphs. We shall refer to directed graphs as digraphs and use the term graph to refer to undirected graphs. A digraph $\Gamma$ is a pair $(V, E)$ of vertices $V$ and edges $E$ where $E \subseteq V \times V$; the digraph $\Gamma$ is said to be finite if $V$ is finite. A graph is a digraph with no edges of the form $(\alpha, \alpha)$ and with the property that $(\alpha, \beta) \in E$ implies $(\beta, \alpha) \in E$. The set of all permutations of $V$ which preserve the adjacency structure of $\Gamma$ is called the automorphism group of $\Gamma$; it is denoted by $\operatorname{Aut}(\Gamma)$. In this paper all digraphs have no loops. For the group-theoretic and graph-theoretic terminology not defined here we refer the reader to $[9,2]$.

Let $G$ be a group and $S$ a subset of $G$ not containing the identity element 1. Recall that the Cayley digraph $\Gamma=\operatorname{Cay}(G, S)$ of $G$ with respect to $S$ has vertex set $G$ and edge set $\{(g, s g) \mid g \in G, s \in S\}$. If $S=S^{-1}$, then $\operatorname{Cay}(G, S)$ can be viewed as an undirected 
graph, identifying an undirected edge with two directed edges $(g, h)$ and $(h, g)$. This graph is called Cayley graph of $G$ with respect to $S$. By a theorem of Sabidussi [8], a digraph $\Gamma$ is a Cayley digraph over a group $G$ if and only if there exists a regular subgroup of Aut $(\Gamma)$ isomorphic to $G$. There is a natural generalization of the Sabidussi's Theorem. A digraph $\Gamma$ is called an $n$-Cayley digraph over a group $G$ if there exists an $n$-orbit semiregular subgroup of $\operatorname{Aut}(\Gamma)$ isomorphic to $G$. 2-Cayley graphs are called by some authors semiCayley graph, see for example [3], and also bi-Cayley graph [5]. Also a special case of 2-Cayley graphs are also called bi-Cayley graph by some authors, see for example [10].

The spectrum of a finite digraph $\Gamma$ is the spectrum of its adjacency matrix $A$, that is, the set of eigenvalues together with their multiplicities. The characteristic polynomial of $\Gamma$ is the characteristic polynomial of $A$, that is the polynomial defined by $\chi_{A}(\lambda)=$ $\operatorname{det}(\lambda I-A)$. It is known that numerous proofs in graph theory depend on the spectrum of graphs and the spectrum of a graph is one of the most important algebraic invariants. The basic relationships between algebraic properties of these eigenvalues and the usual properties of graphs are available in [2].

Lovász [7] showed that determination of the spectrum of a graph with transitive automorphism group can be reduced to the same task for some Cayley graph and found a formula for certain power sums of the eigenvalues in terms of irreducible characters of automorphism group of the graph. Babai [1] succeeded in simplifying Lovasz' formula for the power sums of the eigenvalues of Cayley graphs.

Spectrum of 2-Cayley graphs over abelian groups is computed by Gao and Luo in [3] using matrix theory. It seems that their arguments cannot be extended to non-abelian groups or $n$-Cayaley graphs, $n \geqslant 3$. In this paper we find a factorization of characteristic polynomial of $n$-Cayley digraphs over an arbitrary group in terms of linear representations of the group. We prove that every Cayley graph over a group having a subgroup of index $n$ can be regarded as an $n$-Cayley graph; and compute the spectrum of Caylay graphs over groups having a cyclic subgroup of index 2 . Finally we find the eigenvectors of $n$-Cayley (di)graphs.

\section{Main Results}

In this paper all vector spaces are over the complex field $\mathbb{C}$ and have finite dimension. Throughout the paper $G$ denotes a finite group. We denote by $\mathbb{C}[G]$, the complex vector space of dimension $|G|$. Let $\mathscr{A}=\left\{e_{g} \mid g \in G\right\}$ be an arbitrary basis of $\mathbb{C}[G]$. We identify $\mathbb{C}[G]$ with the vector space of all complex-valued functions on $G$. Thus a function $\varphi: G \rightarrow \mathbb{C}$ corresponds to the vector $\varphi=\sum_{g \in G} \varphi(g) e_{g}$ and vice versa. In particular, the vector $e_{g}$ of the standard basis $\mathscr{A}$ corresponds to a function, also denoted by $e_{g}$, where

$$
e_{g}(h)= \begin{cases}1 & h=g \\ 0 & h \neq g\end{cases}
$$

The (left) regular representation $\rho_{\text {reg }}$ of $G$ on $\mathbb{C}[G]$ is defined by its action on the basis $\left\{e_{h} \mid h \in G\right\}$; that is for all $g, h \in G, \rho_{\text {reg }}(g) e_{h}=e_{g h}$. The regular representation has degree $|G|$. 
Let $\operatorname{Irr}(G)=\left\{\rho_{1}, \ldots, \rho_{m}\right\}$ be the set of all irreducible inequivalent $\mathbb{C}$-representations of $G$. Let $d_{k}$ and $\varrho^{(k)}$ be the degree and a unitary matrix representation of $\rho_{k}, k=1, \ldots, m$, respectively. We keep these notations throughout the paper.

In the following lemma, which seems to be well-known, we construct an orthogonal basis for $\mathbb{C}[G]$ using the matrix representations $\varrho^{(k)}, 1 \leqslant k \leqslant m$.

Lemma 1. Let $\varrho_{i j}^{(k)}(g)$ be the ijth entry of $\varrho^{(k)}(g), 1 \leqslant i, j \leqslant d_{k}$, and $\bar{\varrho}_{i j}^{(k)}=\sum_{g \in G} \overline{\varrho_{i j}^{(k)}(g)} e_{g}$, where $\bar{z}$ denotes the complex conjugate of a complex number $z$. Then

(i) $\left\{\bar{\varrho}_{i j}^{(k)} \mid 1 \leqslant k \leqslant m, 1 \leqslant i, j \leqslant d_{k}\right\}$ form an orthogonal basis for $\mathbb{C}[G]$,

(ii) $\rho_{\text {reg }}(g) \bar{\varrho}_{i j}^{(k)}=\sum_{l=1}^{d_{k}} \varrho_{l i}^{(k)}(g) \bar{\varrho}_{l j}^{(k)}$, for all $g \in G$ and $1 \leqslant i, j \leqslant d_{k}, 1 \leqslant k \leqslant m$,

(iii) $\mathbb{C}[G]=\bigoplus_{k=1}^{m} \bigoplus_{j=1}^{d_{k}} W_{j}^{(k)}$, where $W_{j}^{(k)}=\left\langle\bar{\varrho}_{i j}^{(k)} \mid 1 \leqslant i \leqslant d_{k}\right\rangle$ which is a $\rho_{\text {reg-invariant }}$ subspace of $\mathbb{C}[G]$ of dimension $d_{k}$.

Proof. Let $1 \leqslant k, k^{\prime} \leqslant m$. Then by [9, Corollaries 2, 3, p. 14],

$$
\frac{1}{|G|} \sum_{g \in G} \varrho_{i j}^{(k)}(g) \varrho_{i^{\prime} j^{\prime}}^{\left(k^{\prime}\right)}\left(g^{-1}\right)=\frac{\delta_{i j^{\prime}} \delta_{j i^{\prime}} \delta_{k k^{\prime}}}{d_{k}},
$$

for all $1 \leqslant i, j \leqslant d_{k}$ and $1 \leqslant i^{\prime}, j^{\prime} \leqslant d_{k^{\prime}}$. On the other hand the matrices $\varrho^{(k)}(g)$ are unitary for all $g \in G$ and $1 \leqslant k \leqslant m$ and so $\varrho_{i j}^{(k)}(g)^{-1}=\overline{\varrho_{j i}^{(k)}(g)}$. Hence (1) yields that

$$
\frac{1}{|G|} \sum_{g \in G} \varrho_{i j}^{(k)}(g) \overline{\varrho_{j^{\prime} i^{\prime}}^{\left(k^{\prime}\right)}(g)}=\frac{\delta_{i j^{\prime}} \delta_{j i^{\prime}} \delta_{k k^{\prime}}}{d_{k}} .
$$

Now

$$
\left\langle\bar{\varrho}_{i j}^{(k)}, \bar{\varrho}_{j^{\prime} i^{\prime}}^{\left(k^{\prime}\right)}\right\rangle=\frac{1}{|G|} \sum_{g \in G} \overline{\varrho_{i j}^{(k)}(g)} \varrho_{j^{\prime} i^{\prime}}^{\left(k^{\prime}\right)}(g)=\frac{\delta_{i j^{\prime}} \delta_{j i^{\prime}} \delta_{k k^{\prime}}}{d_{k}},
$$

which shows that $\bar{\varrho}_{i j}^{(k)}, \bar{\varrho}_{j^{\prime} i^{\prime}}^{\left(k^{\prime}\right)}$ are mutually orthogonal vectors of $\mathbb{C}[G]$ and hence independent. On the other hand the cardinality of $\left\{\bar{\varrho}_{i j}^{(k)} \mid 1 \leqslant k \leqslant m, 1 \leqslant i, j \leqslant d_{k}\right\}$ is $\sum_{k=1}^{m} d_{k}^{2}$ which is equal to $|G|$, by $[9$, Corollary 2, p. 18]. Since the dimension of $\mathbb{C}[G]$ as a $\mathbb{C}$-vector space is $|G|$, we conclude that $\left\{\bar{\varrho}_{i j}^{(k)} \mid 1 \leqslant k \leqslant m, 1 \leqslant i, j \leqslant d_{k}\right\}$ is a basis for $\mathbb{C}[G]$. This proves (i).

Now we prove (ii). First note that for every $\varphi \in \mathbb{C}[G]$, and $g, h \in G$ we have $\rho_{\text {reg }}(g) \varphi(h)=\varphi\left(g^{-1} h\right)$, since

$$
\begin{aligned}
\rho_{\mathrm{reg}}(g) \varphi(h) & =\rho_{\mathrm{reg}}(g) \sum_{x \in G} \varphi(x) e_{x}(h) \\
& =\sum_{x \in G} \varphi(x) \rho_{\mathrm{reg}}(g) e_{x}(h)
\end{aligned}
$$




$$
\begin{aligned}
& =\sum_{x \in G} \varphi(x) e_{g x}(h) \\
& =\varphi\left(g^{-1} h\right) .
\end{aligned}
$$

Hence for each $g, h \in G$ we have (here we denote by $[A]_{i j}$ the $i j$ th entry of a matrix A)

$$
\begin{aligned}
\rho_{\text {reg }}(g) \bar{\varrho}_{i j}^{(k)}(h) & =\bar{\varrho}_{i j}^{(k)}\left(g^{-1} h\right) \\
& =\left[\bar{\varrho}^{(k)}(g)^{-1} \bar{\varrho}^{(k)}(h)\right]_{i j} \\
& =\sum_{l=1}^{d_{k}} \varrho_{l i}^{(k)}(g) \bar{\varrho}_{l j}^{(k)}(h) \quad\left(\text { since } \varrho^{(k)}(g) \text { is a unitary matrix }\right)
\end{aligned}
$$

as desired. The statement (iii) is an immediate consequence of (i) and (ii).

If a group $G$ acts on a set $\Omega$ and $\alpha \in \Omega$, we denote by $\alpha^{G}$ and $G_{\alpha}$, the orbit of $G$ on $\Omega$ with representative $\alpha$ and the stabilizer of $\alpha$ in $G$, respectively. Now we will find a useful decomposition of the characteristic polynomial of $n$-Cayley (di)graphs. First we state a very useful and well known equivalent definition of $n$-Cayley (di)graphs.

Lemma 2. A digraph $\Gamma$ is n-Cayley digraph over $G$ if and only if there exist subsets $T_{i j}$ of $G$, where $1 \leqslant i, j \leqslant n$, such that $\Gamma$ is isomorphic to a digraph $X$ with

$$
V(X)=G \times\{1,2, \ldots, n\}, \quad E(X)=\bigcup_{1 \leqslant i, j \leqslant n}\left\{((g, i),(t g, j)) \mid g \in G \text { and } t \in T_{i j}\right\} .
$$

Proof. " $\Rightarrow$ "Let $\alpha_{i}^{G}, 1 \leqslant i \leqslant n$, be the orbits of $G$ on $V(\Gamma)$. Let $T_{i j}:=\{g \in G \mid$ $\left.\left(\alpha_{i}, \alpha_{j}^{g}\right) \in E(\Gamma)\right\}, 1 \leqslant i, j \leqslant n$, and $\varphi: V(\Gamma) \longrightarrow V(X)$, where $\alpha_{i}^{g} \mapsto(g, i)$ and $X$ is defined in (2). We show that $\varphi$ is a digraph isomorphism. Since $V(\Gamma)=\bigcup_{i=1}^{n} \alpha_{i}^{G}$, every vertex $v \in V(\Gamma)$ is of the form $\alpha_{i}^{g}$ for some $g \in G$ and $1 \leqslant i \leqslant n$. Also

$$
\alpha_{i}^{g}=\alpha_{j}^{h} \Longleftrightarrow i=j, g h^{-1} \in G_{\alpha_{i}}(=1) \Longleftrightarrow g=h, i=j .
$$

Hence $\varphi$ is well-defined and one to one. Clearly $\varphi$ is onto. Moreover

$$
\begin{aligned}
\left(\alpha_{i}^{g}, \alpha_{j}^{h}\right) \in E(\Gamma) & \Longleftrightarrow\left(\alpha_{i}, \alpha_{j}^{h g^{-1}}\right) \in E(\Gamma) \\
& \Longleftrightarrow h g^{-1} \in T_{i j} \\
& \Longleftrightarrow((g, i),(h, j)) \in E(X) \\
& \Longleftrightarrow\left(\left(\alpha_{i}^{g}\right)^{\varphi},\left(\alpha_{j}^{h}\right)^{\varphi}\right) \in E(X) .
\end{aligned}
$$

Thus $\varphi$ is a digraph isomorphism as desired.

$" \Leftarrow "$ Let $X$ be the digraph defined in (2). We show that $X$ is an $n$-Cayley digraph over $G$. Consider the action of $G$ on $V(X)$ as $(g, i)^{h}:=(g h, i)$. Let $\varphi$ be the corresponding permutation representation. Then $G^{\varphi} \leqslant \operatorname{Sym}(\mathrm{V}(\mathrm{X}))$. Let $(g, i),(h, j) \in V(X)$. Then for each $a \in G$, we have

$$
((g, i),(h, j)) \in E(X) \Longleftrightarrow h g^{-1} \in T_{i j}
$$




$$
\begin{aligned}
& \Longleftrightarrow(h a)(g a)^{-1} \in T_{i j} \\
& \Longleftrightarrow((g a, i),(h a, j)) \in E(X) \\
& \Longleftrightarrow\left((g, i)^{a^{\varphi}},(h, j)^{a^{\varphi}}\right) \in E(X) .
\end{aligned}
$$

Therefore $G^{\varphi} \leqslant \operatorname{Aut}(X)$. Thus $G \cong G^{\varphi} \leqslant \operatorname{Aut}(X)$ and also $G^{\varphi}$ acts on $V(X)$ semiregularly. Note that $V(X)=\bigcup_{i=1}^{n}(g, i)^{G^{\varphi}}$ is a partition of $V(X)$ to $G^{\varphi}$-orbits for any $g \in G$. Thus the proof is complete.

By Lemma 2, an $n$-Cayley (di)graph is characterized by a group $G$ and $n^{2}$ subsets $T_{i j}$ of $G$ (some subsets may be empty). So we denote an $n$-Cayley (di)graph with respect to $n^{2}$ subsets $T_{i j}$ by $\Gamma=\operatorname{Cay}\left(G ; T_{i j} \mid 1 \leqslant i, j \leqslant n\right)$. Note that this representation is not unique. By Lemma $2, V(\Gamma)=G \times\{1, \ldots, n\},(g, i) \sim(h, j)$ if and only if $h g^{-1} \in T_{i j}$ and $\Gamma$ is undirected if and only if for all $1 \leqslant i, j \leqslant n, T_{i j}=T_{j i}^{-1}$. Note also that $\Gamma$ is a (di)graph without loops if and only if $T_{i i} \subseteq G \backslash\{1\}$, for all $1 \leqslant i \leqslant n$. Let $A=\left[a_{(g, i)(h, j)}\right]_{g, h \in G, 1 \leqslant i, j \leqslant n}$ be the adjacency matrix of $\Gamma$. For a $1 \times m$ vector $v$ and $1 \leqslant i \leqslant n$, we define $v^{i}$ to be a $1 \times n m$ vector with $n$ blocks, whose the $i$ th block is $v$ and other blocks are $0_{1 \times m}$. Let $e_{g}^{i}$ be the $1 \times n|G|$ vector with $n$ blocks, where $i$ th block is $e_{g}$, as defined, and other blocks are $0_{1 \times|G|}$ vectors. Let $V$ be the vector space with basis $\left\{e_{g}^{i} \mid g \in G, 1 \leqslant i \leqslant n\right\}$. Clearly $V \cong \underbrace{\mathbb{C}[G] \oplus \mathbb{C}[G] \oplus \cdots \oplus \mathbb{C}[G]}_{n \text {-times }}$, as $\mathbb{C}[G]=\left\langle e_{g} \mid g \in G\right\rangle$.

So $\operatorname{dim}_{\mathbb{C}} V=n \operatorname{dim}_{\mathbb{C}} \mathbb{C}[G]=n|G|$. Hence we can view $A$ as the linear map

$$
\begin{aligned}
A: V & \rightarrow V \\
e_{g}^{i} & \mapsto \sum_{j=1}^{n} \sum_{h \in G} a_{(h, j)(g, i)} e_{h}^{j}, \quad 1 \leqslant i \leqslant n, g \in G .
\end{aligned}
$$

For an element $g \in G$, we define $\widehat{\rho}_{\text {reg }}(g): V \rightarrow V$ with

$$
e_{h}^{i} \mapsto e_{g h}^{i}, \quad 1 \leqslant i \leqslant n, h \in G .
$$

Then $g \mapsto \widehat{\rho}_{\text {reg }}(g)$ induces a representation $\widehat{\rho}_{\text {reg }}: G \rightarrow G L(V)$.

In the following lemma, we find a relation between $A$ and $\widehat{\rho}_{\text {reg }}$.

Lemma 3. Let $A$ be the adjacency matrix of the digraph $\Gamma=\operatorname{Cay}\left(G ; T_{i j} \mid 1 \leqslant i, j \leqslant n\right)$. For all $1 \leqslant i \leqslant n$ and $g \in G$, we have $A e_{g}^{i}=\sum_{j=1}^{n} \sum_{t \in T_{i j}} \widehat{\rho}_{\text {reg }}(t) e_{g}^{j}$ (with the convention $\sum_{t \in T_{i j}} \widehat{\rho}_{\text {reg }}(t)=0$ if $\left.T_{i j}=\emptyset\right)$.

Proof. We have $a_{(h, j)(g, i)}=1$ if and only if $h g^{-1} \in T_{i j}$. So

$$
\begin{aligned}
A e_{g}^{i} & =\sum_{j=1}^{n} \sum_{h \in G} a_{(h, j)(g, i)} e_{h}^{j} \\
& =\sum_{j=1}^{n} \sum_{\substack{h=t g \\
t \in T_{i j}}} e_{h}^{j}
\end{aligned}
$$

THE ELECTRONiC JOURNAL OF COMBINATORICS 20(3) (2013), \#P57 


$$
\begin{aligned}
& =\sum_{j=1}^{n} \sum_{t \in T_{i j}} e_{t g}^{j} \\
& =\sum_{j=1}^{n} \sum_{t \in T_{i j}} \widehat{\rho}_{\mathrm{reg}}(t) e_{g}^{j}
\end{aligned}
$$

as desired.

For $i=1, \ldots, n$, let $V_{i}$ be a vector space with basis $\left\{e_{g}^{i} \mid g \in G\right\}$. Then for all $h \in G$,

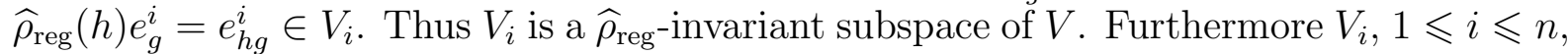
is isomorphic to $\mathbb{C}[G]$ as a $\mathbb{C}$-vector space, $V=\bigoplus_{i=1}^{n} V_{i}$ and $\widehat{\rho}_{\text {reg }}=\left.\bigoplus_{i=1}^{n} \widehat{\rho}_{\text {reg }}\right|_{V_{i}}$. Note that we can identify $V_{i}$ with $\mathbb{C}[G]$ and $\left.\widehat{\rho}_{\text {reg }}\right|_{V_{i}}$ with $\rho_{\text {reg }}$.

If we denote $\left.\widehat{\rho}_{\text {reg }}\right|_{V_{k}}$ by $\widehat{\rho}_{\text {reg, }, k}$, then by part (ii) of Lemma 1 we have the following result.

Lemma 4. Let $\varphi_{1}, \ldots, \varphi_{m}$ be all inequivalent $\mathbb{C}$-irreducible representations of $G$ with degrees $d_{1}, \ldots, d_{m}$, respectively. Let $\varrho^{(l)}$ be a unitary matrix representation of $\varphi_{l}$ and $\bar{\varrho}_{i j}^{(l), k}:=\sum_{g \in G} \overline{\varrho_{i j}^{(l)}(g)} e_{g}^{k}, 1 \leqslant k \leqslant n, 1 \leqslant l \leqslant m$ and $1 \leqslant i, j \leqslant d_{l}$. Then $\widehat{\rho}_{\text {reg }, k}(g) \bar{\varrho}_{i j}^{(l), k}=$ $\sum_{r=1}^{d_{l}} \varrho_{r i}^{(l)}(g) \varrho_{r j}^{(l), k}$.

Proof. First note that $\bar{\varrho}_{i j}^{(l), k}=\sum_{g \in G} \overline{\varrho_{i j}^{(l)}(g)} e_{g}^{k}=\left(\sum_{g \in G} \overline{\varrho_{i j}^{(l)}(g)} e_{g}\right)^{k}=\left(\bar{\varrho}_{i j}^{(l)}\right)^{k}$, where $\bar{\varrho}_{i j}^{(l)}$ is defined in Lemma 1. Now we have

$$
\begin{aligned}
\widehat{\rho}_{\text {reg }, k}(g) \bar{\varrho}_{i j}^{(l), k} & =\widehat{\rho}_{\text {reg, }, k}(g)\left(\bar{\varrho}_{i j}^{(l)}\right)^{k} \\
& =\left(\rho_{\text {reg }}(g) \bar{\varrho}_{i j}^{(l)}\right)^{k} \\
& =\left(\sum_{r=1}^{d_{l}} \varrho_{r i}^{(l)}(g) \bar{\varrho}_{r j}^{(l)}\right)^{k} \\
& \left.=\sum_{r=1}^{d_{l}} \varrho_{r i}^{(l)}(g) \bar{\varrho}_{r j}^{(l), k} \quad \text { (by Lemma } 1(i i)\right)
\end{aligned}
$$

as desired.

For the rest of this section we keep the notations of Lemma 4. Using the notations of this lemma we have the following corollary.

Corollary 5. Let $A$ be the adjacency matrix of digraph $\Gamma=\operatorname{Cay}\left(G ; T_{i j} \mid 1 \leqslant i, j \leqslant n\right)$. Then $A \bar{\varrho}_{i j}^{(l), k}=\sum_{s=1}^{n} \sum_{t \in T_{k s}} \sum_{r=1}^{d_{l}} \varrho_{r i}^{(l)}(t) \bar{\varrho}_{r j}^{(l), s}$.

Proof. We have

$$
A \bar{\varrho}_{i j}^{(l), k}=A\left(\sum_{g \in G} \overline{\varrho_{i j}^{(l)}(g)} e_{g}^{k}\right)
$$




$$
\begin{aligned}
& =\sum_{g \in G} \overline{\varrho_{i j}^{(l)}(g)} A e_{g}^{k} \\
& =\sum_{g \in G} \overline{\varrho_{i j}^{(l)}(g)} \sum_{s=1}^{n} \sum_{t \in T_{k s}} \widehat{\rho}_{\mathrm{reg}}(t) e_{g}^{s} \quad \text { (by Lemma 3) } \\
& =\sum_{s=1}^{n} \sum_{t \in T_{k s}} \sum_{g \in G} \overline{\varrho_{i j}^{(l)}(g)} \widehat{\rho}_{\mathrm{reg}}(t) e_{g}^{s} \\
& =\sum_{s=1}^{n} \sum_{t \in T_{k s}} \sum_{g \in G} \widehat{\rho}_{\mathrm{reg}}(t)\left(\overline{\varrho_{i j}^{(l)}(g)} e_{g}^{s}\right) \\
& \left.=\sum_{s=1}^{n} \sum_{t \in T_{k s}} \sum_{g \in G} \widehat{\rho}_{\mathrm{reg}, s}(t) \overline{\left(\varrho_{i j}^{(l)}(g)\right.} e_{g}^{s}\right) \\
& =\sum_{s=1}^{n} \sum_{t \in T_{k s}} \widehat{\rho}_{\mathrm{reg}, s}(t)\left(\sum_{g \in G} \overline{\varrho_{i j}^{(l)}(g)} e_{g}^{s}\right) \\
& =\sum_{s=1}^{n} \sum_{t \in T_{k s}} \widehat{\rho}_{\mathrm{reg}, s}(t) \bar{\varrho}_{i j}^{(l), s} \\
& \sum_{s}^{n} \sum_{s=1} \sum_{t \in T_{k s}} \varrho_{r=1}^{(l)}(t) \bar{\varrho}_{r j}^{(l), s}(\mathrm{by} \text { Lemma 4) }
\end{aligned}
$$

as desired.

Now we are ready to prove the main result of the paper.

Theorem 6. Let $\Gamma=\operatorname{Cay}\left(G ; T_{i j} \mid 1 \leqslant i, j \leqslant n\right)$ be an $n$-Cayley digraph over a finite group $G$. For each $l \in\{1, \ldots, m\}$, we define $n d_{l} \times n d_{l}$ block matrix $A_{l}:=\left[A_{i j}^{(l)}\right]$, where $A_{i j}^{(l)}=\sum_{t \in T_{j i}} \varrho^{(l)}(t)$. Let $\chi_{A_{l}}(\lambda)$ and $\chi_{A}(\lambda)$ be the characteristic polynomial of $A_{l}$ and $A$, respectively. Then $\chi_{A}(\lambda)=\prod_{l=1}^{m} \chi_{A_{l}}(\lambda)^{d_{l}}$.

Proof. By the notations of Lemma 4 and using Lemma 1, if we put

$$
\mathscr{B}_{j}^{(l), k}=\left\{\bar{\varrho}_{i j}^{(l), k} \mid 1 \leqslant i \leqslant d_{l}\right\} \quad \text { and } V_{j}^{(l), k}=\left\langle\mathscr{B}_{j}^{(l), k}\right\rangle
$$

then $\mathscr{B}^{k}=\bigcup_{l=1}^{m} \bigcup_{j=1}^{d_{l}} \mathscr{B}_{j}^{(l), k}$ is a basis for $V_{k}=\bigoplus_{l=1}^{m} \bigoplus_{j=1}^{d_{l}} V_{j}^{(l), k}$. So

$$
V=\bigoplus_{k=1}^{n} \bigoplus_{l=1}^{m} \bigoplus_{j=1}^{d_{l}} V_{j}^{(l), k}=\bigoplus_{l=1}^{m} \bigoplus_{j=1}^{d_{l}} \bigoplus_{k=1}^{n} V_{j}^{(l), k}
$$

and $\mathscr{B}=\left\{\mathscr{B}^{k} \mid 1 \leqslant k \leqslant n\right\}$ is a basis for $V$. Now put $\mathscr{C}_{j}^{(l)}=\left\{\bar{\varrho}_{i j}^{(l), k} \mid 1 \leqslant k \leqslant n, 1 \leqslant i \leqslant d_{l}\right\}$ and $V_{j}^{(l)}=\left\langle\mathscr{C}_{j}^{(l)}\right\rangle$. Then $V_{j}^{(l)}=\bigoplus_{k=1}^{n} V_{j}^{(l), k}$. Hence $V=\bigoplus_{l=1}^{m} \bigoplus_{j=1}^{d_{l}} V_{j}^{(l)}$. 
On the other hand, by Corollary $5, V_{j}^{(l)}$ is $A$-invariant subspace of $V$ and $\left[\left.A\right|_{V_{j}^{(l)}}\right]_{\mathscr{C}_{j}^{(l)}}=$ $\left[\left.A\right|_{V_{j^{\prime}}^{(l)}}\right]_{\mathscr{C}_{j^{\prime}}^{(l)}}$ for all $1 \leqslant j, j^{\prime} \leqslant d_{l}$, where $[T]_{\mathscr{B}}$ is the matrix of a linear transformation $T$ with respect to the basis $\mathscr{B}$. So by the primary decomposition Theorem, $[A]_{\mathscr{B}}=$ $\operatorname{diag}\left(I_{d_{1}} \otimes A_{1}, I_{d_{2}} \otimes A_{2}, \ldots, I_{d_{m}} \otimes A_{m}\right)$, where $A_{l}=\left[\left.A\right|_{V_{1}^{(l)}}\right]_{\mathscr{C}_{1}^{(l)}}, 1 \leqslant l \leqslant m$. We consider the ordering

$$
\varrho_{1 j}^{(l), 1}, \varrho_{2 j}^{(l), 1}, \ldots, \varrho_{d_{l} j}^{(l), 1}, \varrho_{1 j}^{(l), 2}, \ldots, \varrho_{d_{l} j}^{(l), 2}, \ldots, \varrho_{1 j}^{(l), n}, \ldots, \varrho_{d_{l} j}^{(l), n}
$$

for the elements of $\mathscr{C}_{j}^{(l)}$. Now by Corollary $5, A_{l}=\left[A_{i j}^{(l)}\right]$ is $n d_{l} \times n d_{l}$ block matrix with $d_{l} \times d_{l}$ blocks $A_{i j}^{(l)}, 1 \leqslant i, j \leqslant n$, where $A_{i j}^{(l)}=\sum_{t \in T_{j i}} \varrho^{(l)}(t)$. Now the result is clear.

Let $K_{r, r, \ldots, r}$ be the $n$-partite complete graph. The complement of this graph consists $n$ components isomorphic with the complete graph $K_{r}$. By [2, p. 20], we have

$$
\chi_{K_{r, r, \ldots, r}}(\lambda)=\lambda^{n(r-1)}(\lambda+r(1-n))(\lambda+r)^{n-1} .
$$

Now we derive the latter formula using Theorem 6: To see this, suppose that $G$ is a finite group of order $r$ and $\Gamma=\operatorname{Cay}\left(G ; T_{i j} \mid 1 \leqslant i, j \leqslant n\right)$ where $T_{i i}=\emptyset$ and $T_{i j}=G$, for $i \neq j$. Let $\varrho^{(1)}, \varrho^{(2)}, \ldots, \varrho^{(m)}$ be all inequivalent unitary irreducible representations of $G$. Let $d_{i}$ and $\eta_{i}$ be the degree and character of $\varrho^{(i)}, 1 \leqslant i \leqslant m$, respectively. Also let $\eta_{1}$ be the trivial character. By Theorem $6, \chi_{A}(\lambda)=\Pi_{l=1}^{m} \chi_{A_{l}}(\lambda)^{d_{l}}$, where $A_{l}=\left[A_{i j}^{(l)}\right]_{1 \leqslant i, j \leqslant n}$ and $A_{i j}^{(l)}=\sum_{t \in T_{j i}} \varrho^{(l)}(t)$. By our convention $A_{i i}^{(l)}=0\left(\right.$ since $\left.T_{i i}=\emptyset\right)$. Now let $i \neq j$. Then for every $g \in G$, we have

$$
\varrho^{(l)}(g) A_{i j}^{(l)} \varrho^{(l)}(g)^{-1}=\sum_{t \in G} \varrho^{(l)}(g) \varrho^{(l)}(t) \varrho^{(l)}\left(g^{-1}\right)=\sum_{t \in G} \varrho^{(l)}\left(g t g^{-1}\right)=\sum_{t \in G} \varrho^{(l)}(t)=A_{i j}^{(l)} .
$$

Thus by Schur's Lemma, we have $A_{i j}^{(l)}=\frac{\sum_{g \in G} \eta_{l}(g)}{d_{l}} I_{d_{l}}$. Put $x_{l}=\frac{\sum_{g \in G} \eta_{l}(g)}{d_{l}}$. Then $A_{l}=$ $B_{l} \otimes I_{d_{l}}$, where $B_{l}$ is a circulant $n \times n$ matrix with first row $\left[0, x_{l}, x_{l}, \ldots, x_{l}\right]$. So by [2, p. 16], $\chi_{B_{l}}(\lambda)=\left(\lambda-(n-1) x_{l}\right)\left(\lambda+x_{l}\right)^{n-1}$ and therefore $\chi_{A_{l}}(\lambda)=\left(\lambda-(n-1) x_{l}\right)^{d_{l}}\left(\lambda+x_{l}\right)^{(n-1) d_{l}}$. On the other hand, $x_{1}=|G|$ and $x_{l}=0$ for $l \neq 1$. So

$$
\begin{aligned}
\chi_{A}(\lambda) & =\Pi_{l=1}^{m}\left(\left(\lambda-(n-1) x_{l}\right)^{d_{l}^{2}}\left(\lambda+x_{l}\right)^{(n-1) d_{l}^{2}}\right) \\
& =(\lambda-(n-1)|G|)(\lambda+|G|)^{n-1} \Pi_{l=2}^{m}\left(\lambda^{d_{l}^{2}} \lambda^{(n-1) d_{l}^{2}}\right) \\
& =(\lambda+(1-n)|G|)(\lambda+|G|)^{n-1} \lambda^{n \sum_{l=2}^{m} d_{l}^{2}} \\
& =(\lambda+(1-n)|G|)(\lambda+|G|)^{n-1} \lambda^{n(|G|-1)} .
\end{aligned}
$$

Replacing $|G|=r$, we get $\Gamma \cong K_{r, r, \ldots, r}$ and now the result is clear.

In what follows we present some applications of Theorem 6. It is well-known that the diameter of any connected graph is less than the number of distinct eigenvalues of 
its adjacency matrix (See [2], Corollary 2.7). Thus by Theorem 6 , if $\Gamma$ is a connected $n$-Cayley graph over a finite group $G$, then the diameter of $\Gamma$ is less than $n \sum_{i=1}^{m} d_{i}$, where $d_{1}, \ldots, d_{m}$ are character degrees of $G$. In particular the diameter of any Cayley graph over $G$ is less than sum of character degrees of $G$.

Since an $n$-Cayley (di)graph over a group $G$ is Cayley (di)graph over $G$ if and only if $n=1$, the following corollary is a direct consequence of Theorem 6 .

Corollary 7. (See [4, Corollary 5.3]) Let $\Gamma=\operatorname{Cay}(G, S)$ be a Cayley digraph over a finite group $G$ with irreducible unitary matrix representations $\varrho^{(1)}, \ldots, \varrho^{(m)}$. Let $d_{l}$ be the degree of $\varrho^{(l)}$. For each $l \in\{1, \ldots, m\}$, define a $d_{l} \times d_{l}$ block matrix $A_{l}:=\left[A_{S}^{(l)}\right]$, where $A_{S}^{(l)}=\sum_{s \in S} \varrho^{(l)}(s)$. Let $\chi_{A_{l}}(\lambda)$ and $\chi_{A}(\lambda)$ be the characteristic polynomial of $A_{l}$ and $A$, the adjacency matrix of $\Gamma$, respectively. Then $\chi_{A}(\lambda)=\prod_{l=1}^{m} \chi_{A_{l}}(\lambda)^{d_{l}}$.

Since all irreducible characters of an abelian group have degree 1, Theorem 6 can be applied easily to compute the spectrum of $n$-Cayley (di)graphs over abelian groups. Let $\Gamma=\operatorname{Cay}\left(G, T_{i j} \mid 1 \leqslant i, j \leqslant n\right)$, be an $n$-Cayley (di)graph over a finite abelian group $G$ of order $m$ with irreducible characters $\eta_{1}, \ldots, \eta_{m}$. By Theorem $6, \chi_{A}(\lambda)=\Pi_{l=1}^{m} \chi_{A_{l}}(\lambda)$, where $A_{l}=\left[\sum_{t \in T_{j i}} \eta_{l}(t)\right]_{1 \leqslant i, j \leqslant n}$, which generalizes Corollary 3.3 of [5]. In particular if $n=2$, then $\chi_{A}(\lambda)=\Pi_{l=1}^{m}\left(\lambda-\lambda_{+}^{(l)}\right)\left(\lambda-\lambda_{-}^{(l)}\right)$, where

$$
\lambda_{ \pm}^{(l)}=\frac{c_{11}^{(l)}+c_{22}^{(l)} \pm \sqrt{\left(c_{11}^{(l)}-c_{22}^{(l)}\right)^{2}+4 c_{12}^{(l)} c_{21}^{(l)}}}{2}
$$

and $c_{i j}^{(l)}=\sum_{t \in T_{j i}} \eta_{l}(t)$, which generalizes the main result of [3].

A Cayley digraph over a group with a subgroup of index $n$ is an $n$-Cayley digraph, as the following result shows.

Lemma 8. Let $\Gamma=\operatorname{Cay}(G, S)$ be a Cayley (di)graph. Suppose that there exists a subgroup $H$ of $G$ with index $n$. If $\left\{t_{1}, \ldots, t_{n}\right\}$ is a left transversal to $H$ in $G$, then $\Gamma \cong \operatorname{Cay}\left(H, T_{i j} \mid\right.$ $1 \leqslant i, j \leqslant n)$, where $T_{i j}=\left\{h \in H \mid t_{j}^{-1} h t_{i} \in S\right\}=H \cap t_{j} S t_{i}^{-1}$.

Proof. Let $\Sigma=\operatorname{Cay}\left(H, T_{i j} \mid 1 \leqslant i, j \leqslant n\right)$. Since $\left\{t_{1}, \ldots, t_{n}\right\}$ is a left transversal to $H$ in $G$, every element of $G$ is uniquely expressible in the form $t_{i} h$ with $h \in H$ and $1 \leqslant i \leqslant n$. Define $\psi: G \rightarrow H \times\{1, \ldots, n\}$ where $\left(t_{i} h\right)^{\psi}=(h, i)$. Clearly $\psi$ is a bijection from $V(\Gamma)$ to $V(\Sigma)$. Now $\left(t_{i} h_{1}, t_{j} h_{2}\right) \in E(\Gamma) \Longleftrightarrow t_{j} h_{2} h_{1}^{-1} t_{i} \in S \Longleftrightarrow h_{2} h_{1}^{-1} \in T_{i j} \Longleftrightarrow\left(\left(h_{1}, i\right),\left(h_{2}, j\right)\right) \in$ $E(\Sigma)$. Hence $\psi$ is a (di)graph isomorphism from $\Gamma$ to $\Sigma$.

Corollary 9. Let $\Gamma=\operatorname{Cay}(G, S)$ be a Cayley digraph, $H=\langle a\rangle$ a cyclic subgroup of $G$ of order $n$ and of index 2 with left transversal $\left\{t_{1}, t_{2}\right\}$. Then the characteristic polynomial of the adjacency matrix of $\Gamma$ is $\chi_{A}(\lambda)=\Pi_{k=0}^{n-1}\left(\lambda-\lambda_{k}^{+}\right)\left(\lambda-\lambda_{k}^{-}\right)$, where

$$
\begin{aligned}
& \lambda_{k}^{+}=\frac{\lambda_{k}^{11}+\lambda_{k}^{22}+\sqrt{\left(\lambda_{k}^{11}-\lambda_{k}^{22}\right)^{2}+4 \lambda_{k}^{12} \lambda_{k}^{21}}}{2}, \lambda_{k}^{-}=\frac{\lambda_{k}^{11}+\lambda_{k}^{22}-\sqrt{\left(\lambda_{k}^{11}-\lambda_{k}^{22}\right)^{2}+4 \lambda_{k}^{12} \lambda_{k}^{21}}}{2}, \\
& \lambda_{k}^{i j}=\sum_{t \in T_{j i}} \omega_{n}^{k t} \text { and } T_{i j}=\left\{t \mid 0 \leqslant t \leqslant n-1, a^{t} \in t_{j} S t_{i}^{-1}\right\} .
\end{aligned}
$$


Proof. It is a direct consequence of Lemma 8 and Theorem 6.

Let $\Gamma$ be a $k$-regular graph with $n$ vertices and adjacency matrix $A$. Let $A^{c}$ be the adjacency matrix of the complement of $\Gamma$. Then $(\lambda+k+1) \chi_{A^{c}}(\lambda)=(-1)^{n}(\lambda-n+$ $k+1) \chi_{A}(-\lambda-1)$, see [2, p. 20]. Clearly Cayley graphs are regular. If $n \geqslant 2, n-$ Cayley graphs are not necessarily regular, but we have a similar relation between the characteristic polynomials of any $n$-Cayley graph and its complement which is given in the next theorem.

Theorem 10. Let $\Gamma=\operatorname{Cay}\left(G, T_{i j} \mid 1 \leqslant i, j \leqslant n\right)$ be an $n$-Cayley graph over a finite group $G$. Let $\Gamma^{c}$ be the complement of $\Gamma$ with adjacency matrix $A^{c}$. Then the characteristic polynomials of $\Gamma$ and $\Gamma^{c}$ are related with the following equation:

$$
\chi_{B_{1}}(\lambda) \chi_{A}(-\lambda-1)=(-1)^{|G|-1} \chi_{A_{1}}(-\lambda-1) \chi_{A^{c}}(\lambda),
$$

where $B_{1}=|G| J-I_{n}-A_{1}, J$ is the all ones matrix of degree $n$, and $A_{1}=\left[\left|T_{j i}\right|\right]_{1 \leqslant i, j \leqslant n}$.

Proof. Since $\operatorname{Aut}(\Gamma)=\operatorname{Aut}\left(\Gamma^{c}\right), \Gamma^{c}$ is an $n$-Cayley graph over $G$. Furthermore $\Gamma^{c}=$ $\operatorname{Cay}\left(G, S_{i j} \mid 1 \leqslant i, j \leqslant n\right)$, where $S_{i i}=G \backslash\left(T_{i i} \cup\{1\}\right)$ and $S_{i j}=G \backslash T_{i j}$, where $i \neq j$. By Theorem 6, $\chi_{A^{c}}(\lambda)=\prod_{l=1}^{m} \chi_{B_{l}}(\lambda)^{d_{l}}$, where $B_{l}=\left[B_{i j}^{(l)}\right]$ is an $n d_{l} \times n d_{l}$ matrix and $B_{i j}^{(l)}=\sum_{s \in S_{j i}} \varrho^{(l)}(s)$ and $\chi_{A}(\lambda)=\prod_{l=1}^{m} \chi_{A_{l}}(\lambda)^{d_{l}}$ as $A_{l}$ defined in Theorem 6 .

For $i \neq j$ we have

$$
B_{i j}^{(l)}=\sum_{s \in S_{j i}} \varrho^{(l)}(s)=\sum_{x \in G} \varrho^{(l)}(x)-\sum_{t \in T_{j i}} \varrho^{(l)}(t)
$$

and for $i=j$ we have

$$
B_{i i}^{(l)}=\sum_{x \in G} \varrho^{(l)}(x)-\sum_{t \in T_{i i}} \varrho^{(l)}(t)-I_{d_{l}}
$$

Put $X_{l}=\sum_{x \in G} \varrho^{(l)}(x)$. Then for every $g \in G$, we have

$$
\varrho^{(l)}(g) X_{l} \varrho^{(l)}(g)^{-1}=\sum_{x \in G} \varrho^{(l)}(g) \varrho^{(l)}(x) \varrho^{(l)}\left(g^{-1}\right)=\sum_{x \in G} \varrho^{(l)}\left(g x g^{-1}\right)=\sum_{x \in G} \varrho^{(l)}(x)=X_{l} .
$$

Therefore by Schur's Lemma, we have $X_{l}=\frac{\sum_{g \in G} \eta_{l}(g)}{d_{l}} I_{d_{l}}$. Hence $X_{1}=|G|$ and $X_{l}=0_{d_{l}}$ for $l \neq 1$, where $0_{d_{l}}$ is the $d_{l} \times d_{l}$ zero matrix. Therefore for all $l \neq 1$,

$$
B_{l}=-A_{l}-\operatorname{diag}\left(I_{d_{l}}, I_{d_{l}}, \ldots, I_{d_{l}}\right),
$$

and $B_{1}=|G| J-I_{n}-A_{1}$, where $J$ is the all ones matrix of degree $n$. Furthermore if $l \neq 1$ then $\chi_{B_{l}}(\lambda)=\prod_{\mu \in \operatorname{Spec}\left(A_{l}\right)}(\lambda+\mu+1)$. So

$$
\chi_{A^{c}}(\lambda)=\chi_{B_{1}}(\lambda) \prod_{l=2}^{m} \prod_{\mu \in \operatorname{Spec}\left(A_{l}\right)}(\lambda+\mu+1)^{d_{l}} .
$$


Also we have

$$
\begin{aligned}
\chi_{A}(-\lambda-1) & =\chi_{A_{1}}(-\lambda-1) \prod_{l=2}^{m} \chi_{A_{l}}(-\lambda-1)^{d_{l}} \\
& =\chi_{A_{1}}(-\lambda-1) \prod_{l=2}^{m} \prod_{\mu \in \operatorname{Spec}\left(A_{l}\right)}(-\lambda-1-\mu)^{d_{l}} \\
& =(-1)^{d_{2}^{2}+d_{3}^{2}+\cdots+d_{m}^{2}} \chi_{A_{1}}(-\lambda-1) \prod_{l=2}^{m} \prod_{\mu \in \operatorname{Spec}\left(A_{l}\right)}(\lambda+1+\mu)^{d_{l}} \\
& =(-1)^{|G|-1} \chi_{A_{1}}(-\lambda-1) \prod_{l=2}^{m} \prod_{\mu \in \operatorname{Spec}\left(A_{l}\right)}(\lambda+1+\mu)^{d_{l}}
\end{aligned}
$$

which implies that $\chi_{B_{1}}(\lambda) \chi_{A}(-\lambda-1)=(-1)^{|G|-1} \chi_{A_{1}}(-\lambda-1) \chi_{A^{c}}(\lambda)$ as desired.

\section{Eigenvectors of $n$-Cayley (di)graphs}

In this section we determine the corresponding eigenspace of each eigenvalue of $n$-Cayley digraph $\Gamma$. We use the notations of Theorem 6 .

Lemma 11. Let $v_{(k)}=\left(v_{1}, \ldots, v_{n}\right)$ be an eigenvector of $A_{k}, 1 \leqslant k \leqslant m$, associated with $\lambda$. Then the following vectors are distinct linearly independent $d_{k}$ eigenvectors of digraph $\Gamma$ associated with $\lambda$ :

$$
v_{(k)}^{j}:=\sum_{s=1}^{n} \sum_{g \in G}\left[v_{s} \cdot \bar{\varrho}_{j}^{(k)}(g)\right] e_{g}^{s}, \quad 1 \leqslant j \leqslant d_{k}
$$

where $\cdot$ is the usual inner product and $\bar{\varrho}_{j}^{(k)}(g)$ is a vector whose coordinates are the complex conjugate of the coordinates of $j$ th column of $\varrho^{(k)}(g)$.

Proof. By Corollary 5, we have $A \bar{\varrho}_{i j}^{(l), k}=\sum_{s=1}^{n} \sum_{t \in T_{k s}} \sum_{r=1}^{d_{l}} \varrho_{r i}^{(l)}(t) \bar{\varrho}_{r j}^{(l), s}$. For $i=1,2, \ldots, n$, let $v_{i}=\left(v_{i 1}, v_{i 2}, \ldots, v_{i d_{k}}\right)$. Then

$$
\begin{aligned}
v_{(k)}^{j} & =\sum_{s=1}^{n} \sum_{g \in G}\left[v_{s} \cdot \bar{\varrho}_{j}^{(k)}(g)\right] e_{g}^{s} \\
& =\sum_{s=1}^{n} \sum_{t=1}^{d_{k}} v_{s t} \sum_{g \in G} \overline{\varrho_{t j}^{(k)}(g)} e_{g}^{s} \\
& =\sum_{s=1}^{n} \sum_{t=1}^{d_{k}} v_{s t} \bar{\varrho}_{t j}^{(k), s} .
\end{aligned}
$$


Now we have

$$
\begin{aligned}
A v_{(k)}^{j} & =\sum_{s=1}^{n} \sum_{t=1}^{d_{k}} v_{s t} A \bar{\varrho}_{t j}^{(k), s} \\
& =\sum_{s=1}^{n} \sum_{t=1}^{d_{k}} v_{s t} \sum_{s^{\prime}=1}^{n} \sum_{t^{\prime} \in T_{s s^{\prime}}} \sum_{r=1}^{d_{k}} \varrho_{r t}^{(k)}\left(t^{\prime}\right) \bar{\varrho}_{r j}^{(k), s} \quad \text { (by Corollary 5) } \\
& =\sum_{s=1}^{n} \sum_{r=1}^{d_{k}}\left[\sum_{t=1}^{d_{k}} \sum_{s^{\prime}=1}^{n} \sum_{t^{\prime} \in T_{s s^{\prime}}} v_{s t} \varrho_{r t}^{(k)}\left(t^{\prime}\right)\right] \bar{\varrho}_{r j}^{(k), s} \\
& =\sum_{s=1}^{n} \sum_{r=1}^{d_{k}} \lambda v_{s r} \bar{\varrho}_{r j}^{(k), s} \\
& =\lambda \sum_{s=1}^{n} \sum_{r=1}^{d_{k}} v_{s r} \bar{\varrho}_{r j}^{(k), s} \\
& =\lambda v_{(k)}^{j}
\end{aligned}
$$

as desired. Since $\left\{\bar{\varrho}_{i j}^{(k), s} \mid 1 \leqslant k \leqslant m, 1 \leqslant s \leqslant n, 1 \leqslant i, j \leqslant d_{k}\right\}$ is an orthogonal basis of $V$ (the corresponding vector space of the adjacency matrix $A), v_{(k)}^{j}$ 's are distinct and linearly independent.

An eigenvector of the adjacency matrix of a graph $\Gamma$ is said to be main eigenvector if it is not orthogonal to the all ones vector $\mathbf{j}$. An eigenvalue of a graph $\Gamma$ is said to be a main eigenvalue if it has a main eigenvector. By Perron-Frobenius Theorem, the largest eigenvalue of a graph is a main eigenvalue. It is also well known that a graph is regular if and only if it has exactly one main eigenvalue. So for every Cayley graph $\Gamma=\operatorname{Cay}(G, S)$, $|S|$ is the only main eigenvalue of $\Gamma$. Since $n$-Cayley graphs, for $n \geqslant 2$ are not necessarily regular, determining the main eigenvalues of these graphs seems to be important. In the following corollary we determine the main eigenvalues of $n$-Cayley graphs.

Corollary 12. Let $\Gamma=\operatorname{Cay}\left(G, T_{i j} \mid 1 \leqslant i, j \leqslant n\right)$ be an $n$-Cayley graph over a finite group $G$ and $n \geqslant 2$. The main eigenvalues of $\Gamma$ is equal to main eigenvalues of the matrix $A_{1}=\left[\left|T_{j i}\right|\right]_{1 \leqslant i, j \leqslant n}$.

Proof. Using the notations of Lemma 11, we have

$$
\begin{aligned}
v_{(k)}^{j} \cdot \mathbf{j} & =\left(\sum_{s=1}^{n} \sum_{g \in G}\left[v_{s} \cdot \bar{\varrho}_{j}^{(k)}(g)\right] e_{g}^{s}\right) \cdot \mathbf{j} \\
& =\sum_{s=1}^{n} \sum_{g \in G}\left[v_{s} \cdot \bar{\varrho}_{j}^{(k)}(g)\right] e_{g}^{s} \cdot \mathbf{j} \\
& =\sum_{s=1}^{n} \sum_{g \in G}\left[v_{s} \cdot \bar{\varrho}_{j}^{(k)}(g)\right]
\end{aligned}
$$




$$
=\sum_{s=1}^{n} v_{s} \cdot\left[\sum_{g \in G} \bar{\varrho}_{j}^{(k)}(g)\right]
$$

Also $\sum_{g \in G} \bar{\varrho}_{j}^{(k)}(g)$ is the complex conjugate of $j$ th column of $\sum_{g \in G} \varrho^{(k)}(g)$. On the other hand, by Schur's Lemma $\sum_{g \in G} \varrho^{(1)}(g)=|G|$ and for all $k \neq 1, \sum_{g \in G} \varrho^{(k)}(g)=0_{d_{k}}$, where $0_{d_{k}}$ is the all zeros matrix of order $d_{k}$. This implies that

$$
v_{(k)}^{j} \cdot \mathbf{j}= \begin{cases}0 & k \neq 1 \\ |G| \sum_{s=1}^{n} v_{s} & k=1\end{cases}
$$

Since $v_{(1)}^{j} \cdot \mathbf{j}=|G| \sum_{s=1}^{n} v_{s}=|G|\left(v_{(1)} \cdot \mathbf{j}^{\prime}\right)$, where $\mathbf{j}^{\prime}$ is the all ones vector $1 \times n$, the result is clear.

Corollary 13. Let $\Gamma=\operatorname{Cay}\left(G, T_{i j} \mid 1 \leqslant i, j \leqslant 2\right)$ be a 2-Cayley graph over a group $G$. Then $\Gamma$ has exactly two main eigenvalues if and only if $\left|T_{11}\right| \neq\left|T_{22}\right|$.

Proof. Let $\Gamma$ has exactly two main eigenvalues. If $\left|T_{11}\right|=\left|T_{22}\right|$, then $\Gamma$ is regular and by [6, Proposition 1.4], $\Gamma$ must have exactly one main eigenvalue which is a contradiction. Conversely, let $\left|T_{11}\right| \neq\left|T_{22}\right|$. Then $\Gamma$ is not regular. Also by Corollary $12, \Gamma$ has at most two main eigenvalues and by Perron-Frobenius Theorem, the largest eigenvalue of $\Gamma$ is a main eigenvalue. So by [6, Proposition 1.4], $\Gamma$ has exactly two main eigenvalues.

Corollary 14. Let $\Gamma=\operatorname{Cay}\left(G, T_{i j} \mid 1 \leqslant i, j \leqslant 2\right)$ be a 2-Cayley graph over a group $G$. If $\left|T_{11}\right| \neq\left|T_{22}\right|$ then $\Gamma$ has exactly two orbits on $V(\Gamma)$ which are the same orbits of $R_{G}$.

Proof. Let $\left|T_{11}\right| \neq\left|T_{22}\right|$. By Corollary 13, $\Gamma$ has exactly two main eigenvalues. On the other hand, $A:=\operatorname{Aut}(\Gamma)$ has at least two orbits on $V(\Gamma)$, say $\alpha^{A}$ and $\beta^{A}$. Let $O_{1}=\alpha^{R_{G}}$ and $O_{2}=\beta^{R_{G}}$ be two orbits of $R_{G}$ then $O_{1} \subseteq \alpha^{A}$ and $O_{2} \subseteq \beta^{A}$. Hence $O_{1} \cap O_{2}=\emptyset$ and so $O_{1} \cup O_{2}=V=\alpha^{A} \cup \beta^{A}$. This shows that $\Gamma$ has exactly two distinct orbits which are the same orbits of $R_{G}$.

Note that the converse of the above corollary is not true. To see this, consider the generalized Peterson graph $\Gamma=P(h, t)$, where $t^{2} \neq 1(\bmod h)$. Then $P(h, t)$ is not vertex-transitive (see [2, pp. 104, 105]), and so as we proved in the above corollary, $\Gamma$ has two orbits on $V(\Gamma)$ and is a 2-Cayley graph over a cyclic group $\langle a\rangle$ of order $h$, where $T_{11}=\left\{a, a^{-1}\right\}, T_{22}=\left\{a^{t}, a^{-t}\right\}$ and $T_{12}=T_{21}=\{1\}$.

\section{References}

[1] L. Babai. Spectra of Cayley graphs. J. Comb. Theory Ser. B, 27: 180-189, 1979.

[2] N. Biggs. Algebraic Graph Theory. Cambridge University Press, 1974.

[3] X. Gao and Y. Luo. The spectrum of semi-Cayley graphs over abelian groups. Linear Algebra Appl., 432: 2974-2983, 2010. 
[4] P. Kaski. Eigenvectors and spectra of Cayley graphs. Manuscript for a seminar given at Helsinki University of Technology, 2002.

[5] I. Kovács, A. Malnič, D. Marušič and Š. Miklavič. Transitive group actions: (im)primitivity and semiregular subgroups. arXiv:math/0701686, 2007.

[6] P. Rowlinson. The main eigenvalues of a graph: a survey. Appl. Anal. Discrete Math. 1: 445-471, 2007.

[7] L. Lovász. Spectra of graphs with transitive groups. Periodica Math. Hung. 6(2): 191-195, 1975.

[8] G. Sabidussi. Vertex-transitive graphs. Monatsh. Math. 68: 426-438, 1964.

[9] J. -P. Serre. Linear representations of finite groups. Graduate Texts in Mathematics 42, Springer-Verlag, New York, 1997.

[10] X. Zou and J. X. Meng. Some algebraic properties of Bi-Cayley graphs. Acta Math. Sin. (Chin. Ser.) 50 (5): 1075-1080, 2007. 